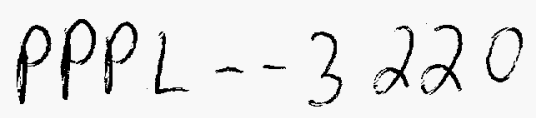

F1-CN-64/D2-2

\title{
3D SIMULATION STUDIES of TOKAMAK PLASMAS using MHD and EXTENDED-MHD MODELS
}

\author{
W. PARK, Z. CHANG, E. FREDRICKSON, G.Y. FU, N. POMPHREY \\ Princeton University Plasma Physics Laboratory, Princeton, New Jersey \\ H.R. STRAUSS \\ New York University, New York, New York \\ L.E. SUGIYAMA \\ Massachusetts Institute of Technology, Cambridge, Massachusetts \\ USA
}

\section{DISCLAIMER}

This report was prepared as an account of work sponsored by an agency of the United States Government. Neither the United States Government nor any agency thereof, nor any of their employees, makes any warranty, express or implied, or assumes any legal liability or responsibility for the accuracy, completeness, or usefulness of any information, apparatus, product, or process disclosed, or represents that its use would not infringe privately owned rights. Reference herein to any specific commercial product, process, or service by trade name, trademark, manufacturer, or otherwise does not necessarily constitute or imply its endorsement, recommendation, or favoring by the United States Government or any agency thereof. The views and opinions of authors expressed herein do not necessarily state or reflect those of the United States Government or any agency thereof. 


\section{DISCLAIMER}

Portions of this document may be illegible in electronic image products. Images are produced from the best available original document. 
Abstract

The M3D(Multi-level 3D) tokamak simulation project aims at the simulation of tokamak plasmas using a multi-level tokamak code package. Several current applications using MHD and Extended-MHD models are presented; high- $\beta$ disruption studies in reversed shear plasmas using the MHD level MH3D code, $\omega_{* i}$ stabilization and nonlinear island rotation studies using the two-fluid level MH3D-T code, studies of nonlinear saturation of TAE modes using the hybrid particle/MHD level $M H 3 D-K$ code, and unstructured mesh $M H 3 D^{++}$code studies. In particular, three internal mode disruption mechanisms are identified from simulation results which agree well with experimental data.

\section{Introduction}

Recent nonlinear MHD simulation results for high- $\beta$ disruptions[1] and double tearing sawteeth[2] have reaffirmed that many global behaviors of tokamak plasmas can be successfully explained using MHD simulation. However, as tokamak experiments reach higher performance regimes, more sophisticated experimental diagnostics coupled with ever expanding computer capabilities have increased both the need for and the feasibility of 3D nonlinear global simulations using models more realistic than MHD. We currently use various "ExtendedMHD" models as well as the MHD model to study the global behavior of tokamak plasmas. These include a two-fluid model which is used to study finite gyroradius drift-MHD modes, and a Particle/MHD hybrid model which is used to study the nonlinear evolution of kinetic-MHD modes. These and the unstructured mesh capability represent the present status of our M3D(Multi-level 3D) tokamak simulation project.

The M3D project aims at the simulation of tokamak plasmas using a multilevel tokamak code package. A multi-level code is necessary for the study of tokamaks, where complex phenomena can be modeled with various levels of realism. By comparing results from different levels, one can delineate the physics involved and ensure that particular approximations are relevant. This is also a step by step path which leads toward a comprehensive tokamak simulation code which would include most of the relevant physics and also allow various option levels in complexity of physics and geometry. A higher level M3D code contains the lower level codes, such that lower level benchmarks are still useful and the simulation can change to a different level at any point in the calculation. (A simulation code with complete physics, but without intermediate option levels, would produce results too complex for the user to delineate the physics involved and would be very difficult to benchmark completely.)

In the following sections, we present $3 \mathrm{D}$ simulation studies using various option levels of the M3D project; high- $\beta$ disruption studies in reversed shear plasmas using the MHD level $M H 3 D$ code, $\omega_{* i}$ stabilization and nonlinear island rotation studies using the two-fluid level $M H 3 D-T$ code, studies of nonlinear saturation of TAE modes using the hybrid particle/MHD level $M H 3 D-K$ code, and unstructured mesh $M H 3 D^{++}$code studies. 


\section{MHD simulation}

The MH3D code is used to study the mechanisms of internal mode disruption in reversed shear plasmas. Previous studies of normal shear plasmas have shown that toroidally localized high-n ballooning modes can be driven unstable by local pressure steepening in the bad curvature region, which arises from the evolution of low-n modes.[1] Nonlinearly, the high-n mode becomes even more localized and produces a strong local pressure bulge which destroys the flux surfaces resulting in a thermal quench.

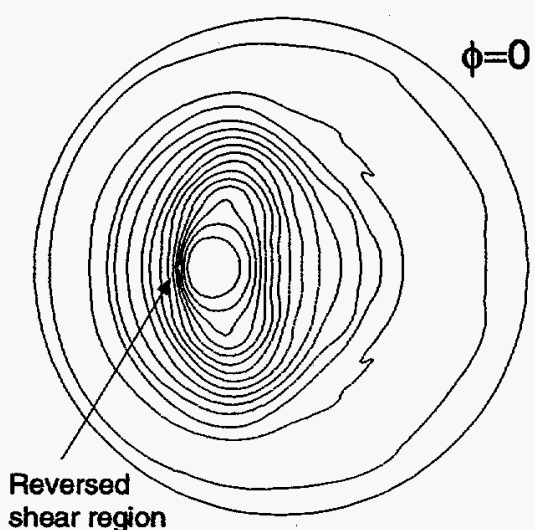

(a)
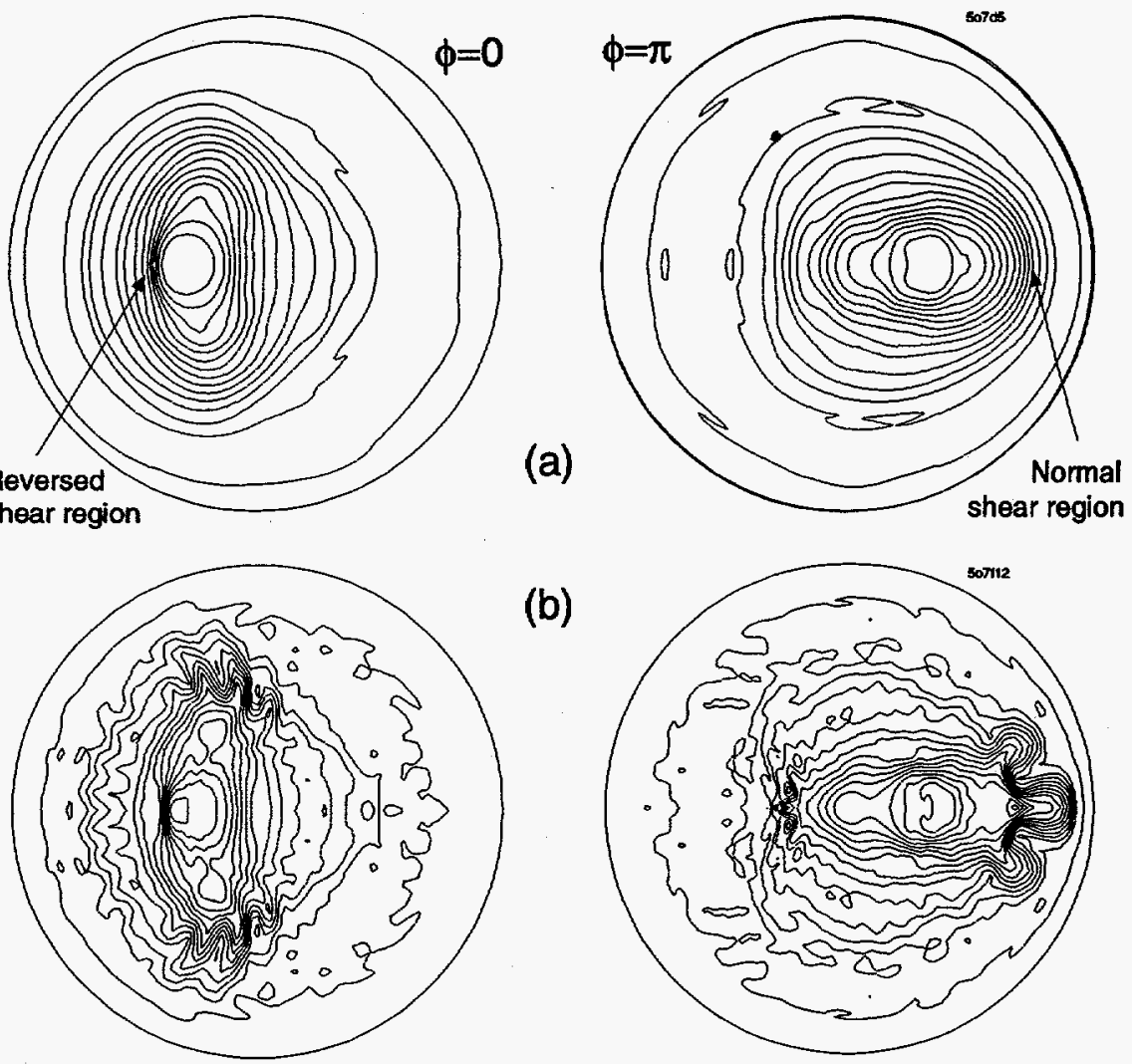

(b)

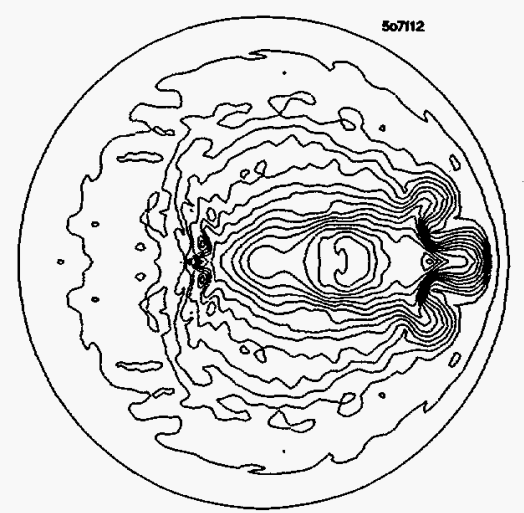

Figure 1: (a)Pressure contours of the 3D equilibrium. Two local pressure steepenings occur; the stronger one inside the reversed shear core region at $\phi=0$, and the other in the normal shear region at $\phi=\pi$. (b)The later nonlinear development of the pressure. The localized high-n ballooning mode develops only from the pressure steepening in the normal shear region.

A similar behavior is also seen in simulations of reversed shear plasmas. The Fig. 1(a) shows pressure contours of a 3D equilibrium which results from the nonlinear evolution of an $n=1$ linear instability using a TFTR initial profile with $3.8 \%$ peak $\beta$. (The aspect ratio used is 2.9 , but a smaller aspect ratio is depicted in the figure only to save space.) This $3 \mathrm{D}$ equilibrium has two local pressure steepenings both on the outboard side as indicated with arrows; the stronger one inside the reversed shear core region at toroidal angle $\phi=0$, and the other in the normal shear region at $\phi=\pi$. A toroidally localized high-n ballooning mode grows out of the local steep pressure region in the normal shear region and eventually destroys the flux surfaces resulting in a thermal quench, as shown in Fig. 1(b). The toroidally localized steep pressure gradient inside the reversed 
shear core, although much stronger than the one outside, remains stable, showing that the advantage of the reversed shear profile regarding pressure driven modes extends far into the 3D configuration.
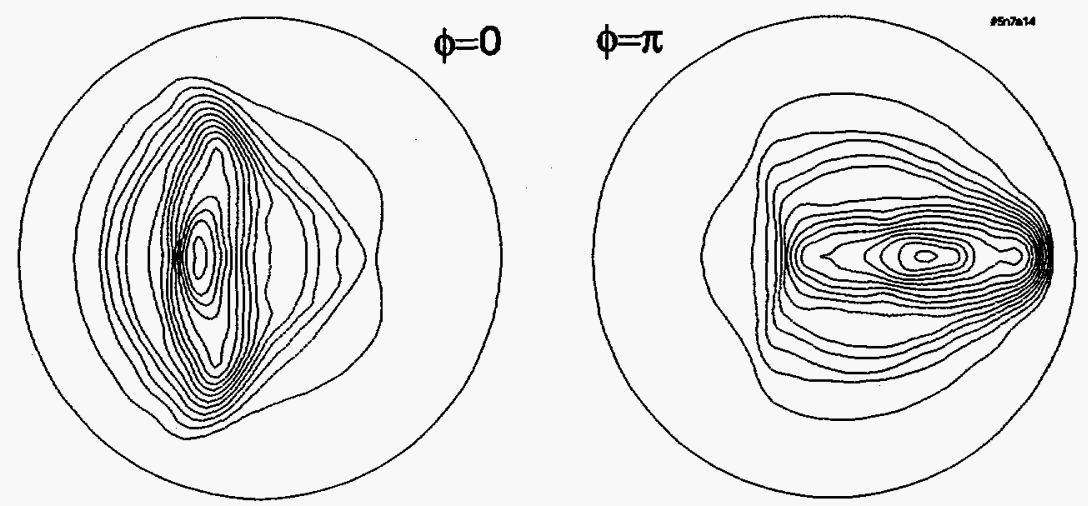

Figure 2: The nonlinear deformation of pressure contours evolving from an $m=2$ dominant $\mathrm{n}=1$ mode, when toroidally localized high- $\mathrm{n}$ modes are stable.

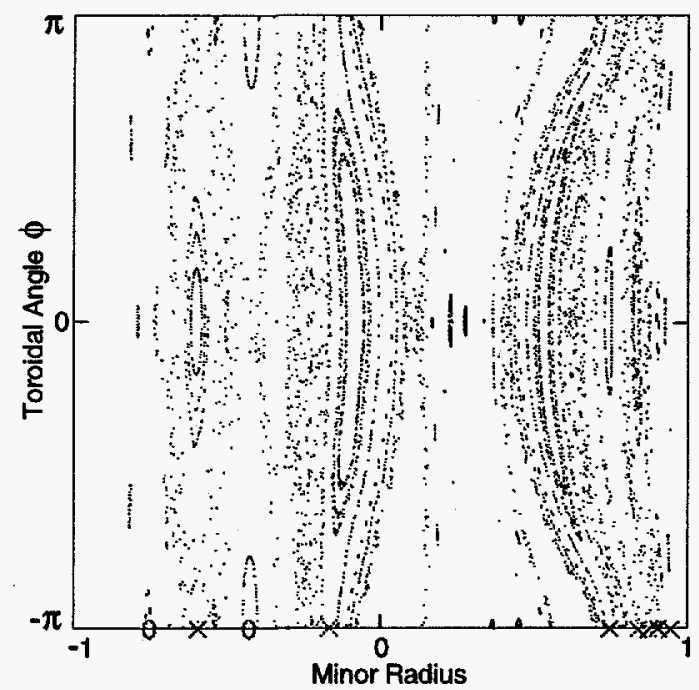

Figure 3: Puncture plot of field lines on the toroidal mid-plane of the torus.

For reversed shear cases, disruptions can also be caused by low- $n$ modes alone without a toroidally localized high-n ballooning mode, when the $q_{\min }$ is close enough or lower than 2. (This will probably also apply to $q_{\min }$ close to other rational numbers like 3.) Fig. 2 shows the nonlinear deformation of pressure evolving from an $m=2$ dominant $n=1$ mode. In this case, a toroidally localized high-n ballooning mode is not destabilized, probably because the $q$ profile used has a smaller flat region around $q_{\min }$ compared to the previous case. The pressure bulge shown on the right figure pushes the plasma into the plasma boundary and drives magnetic reconnection until the outer region becomes stochastic.

This is seen in Fig. 3 which shows the puncture plot of field lines on the toroidal mid-plane of the torus. The pressure bulge shown on the right figure of Fig. 2 corresponds to the bulge on the right side of the $\phi=-\pi$ line(which is the same line as $\phi=\pi$ ). This bulge drives reconnection producing aligned 
$X$-points of various island chains as indicated by $X^{\prime} s$ on the figure, and causes stochasticity. On the inboard side, mixed $X$-points and $O$-points occur as shown by $X^{\prime} s$ and $O^{\prime} s$ on the left side of the $\phi=-\pi$ line. (This mechanism is similar to the pressure bulge in $\mathrm{m}=1$ reconnection cases.[3])

The above two mechanisms of internal mode disruption are due to high- $\beta$ effects. Another mechanism which can cause disruption for a reversed shear plasma is when a double-tearing reconnection occurs with a large mixing radius.[2] This can happen for both high- $\beta$ and low- $\beta$ plasmas. In summary, three mechanisms are identified that can cause an internal mode disruption in high temperature reversed shear plasmas; (1)toroidally localized high-n modes driven unstable by local pressure steepening in the bad curvature region that arises from the evolution of low-n modes, (2)a large pressure bulge caused by the nonlinear development of a low-n mode, (3)a double-tearing reconnection with a large mixing radius. The mechanism (3) can occur only when $q_{\min }$ is lower than 2(or other low mode number rational surfaces), while mechanisms (1) and (2) do not have such a restriction. All three mechanisms are seen in the experiment and the experimental data agrees well with the scenarios presented here. In addition, the mechanism (3) can be mixed with either of the other two mechanisms to produce a disruption.

\section{Two-fluid simulation}

The $M H 3 D$ - $T$ code[4] is the two-fluid extension of the $M H 3 D$ code. The twofluid equations are obtained by generalizing the perturbative drift ordering[5] to arbitrary perturbation size. They are closely related, although not identical, to the collisional Braginskii equations [6]. The model was chosen, in part, to transform smoothly into the resistive MHD equations in the limit of vanishing gyroradii.

The drift ordering [5] assumes fluid velocities and growth rates small compared to the thermal velocity scales of the MHD ordering, $v / v_{t h} \sim \delta, \partial / \partial t \sim$ $\delta v_{t h} / L$, and $\omega / \Omega_{c i} \sim \rho_{i} / L \sim \delta$, where $\delta$ is a characteristic small parameter, $v_{t h}$ is the ion thermal speed, $L$ a characteristic equilibrium scale length, $\omega$ a typical frequency, $\Omega_{c i}$ the ion cyclotron frequency, and $\rho_{i}$ the ion gyroradius. The ordering introduces the diamagnetic velocities

$$
\begin{aligned}
\mathbf{v}_{* j} & =\mathbf{B} \times \nabla p_{j} /\left(q_{j} n_{j} B^{2}\right) \\
\mathbf{v}_{* T j} & =\mathbf{B} \times \nabla T_{j} /\left(q_{j} B^{2}\right),
\end{aligned}
$$

for $j=e, i$, where $q_{j}$ is the particle charge.

The fluid velocities can be written exactly as

$$
\begin{aligned}
\mathbf{v}_{i} & =\mathbf{v}+\mathbf{v}_{d i} \\
\mathbf{v}_{e} & =\mathbf{v}+\mathbf{v}_{* e}-\mathbf{J}_{\|} / e n_{e} \\
\mathbf{v} & =\mathbf{v}_{\perp}+\mathbf{v}_{i \|},
\end{aligned}
$$

where $\mathbf{v}_{\perp}$ is the perpendicular guiding center velocity of the electrons and ions, neglecting magnetic drifts. The generalized "diamagnetic" part $\mathbf{v}_{d i}$ of the ion fluid velocity perpendicular to the magnetic field is defined to be

$$
\mathbf{v}_{d i} \equiv \frac{\mathbf{J}_{\perp}}{e n_{e}}+\mathbf{v}_{* e}
$$


where $\mathbf{v}_{* e}$ is given by Eq. (1) with $j=e$.

In rationalized emu units, the essential features of our two-fluid model can be summarized as

$$
\begin{aligned}
\frac{\partial \mathbf{v}}{\partial t}+(\mathbf{v} \cdot \nabla) \mathbf{v} & =-\left(\dot{\mathbf{v}_{d i}} \cdot \nabla\right) \mathbf{v}_{\perp}+\frac{\mathbf{J} \times \mathbf{B}}{n m_{i}}-\frac{\nabla p}{n m_{i}}+\mu \nabla^{2} \mathbf{v}_{i} \\
\frac{\partial \mathbf{B}}{\partial t} & =-\nabla \times \mathbf{E} \\
\mathbf{E}+\mathbf{v} \times \mathbf{B}= & \eta \mathbf{J}-\frac{\nabla_{\|} p_{e}}{e n} \\
\frac{\partial p_{i}}{\partial t}+\mathbf{v}_{i} \cdot \nabla p_{i} & =-\Gamma_{i} p_{i} \nabla \cdot \mathbf{v}_{i}+\nabla \cdot n \kappa_{\perp i} \nabla_{\perp}\left(\frac{p_{i}}{n}\right)+\nabla \cdot n \kappa_{\| i} \nabla_{\|}\left(\frac{p_{i}}{n}\right) \\
\frac{\partial p_{e}}{\partial t}+\mathbf{v}_{e} \cdot \nabla p_{e}= & -\Gamma_{i} \nabla \cdot\left(p_{i} \mathbf{v}_{* T i}\right) \\
\frac{\partial n_{e}}{\partial t}+\mathbf{v}_{e} \cdot \nabla n \cdot \mathbf{v}_{e}+\nabla \cdot n \kappa_{\perp e} \nabla_{\perp}\left(\frac{p_{e}}{n}\right)+\nabla \cdot n \kappa_{\| e} \nabla_{\|}\left(\frac{p_{e}}{n}\right) & -\Gamma_{e} \nabla \cdot\left(p_{e} \mathbf{v}_{* T e}\right) \\
\mathbf{J}= & \nabla \times \mathbf{B} \\
0= & \nabla \cdot \mathbf{B} .
\end{aligned}
$$

The electron mass is neglected and quasineutrality, $n_{e}=n_{i}=n$, is assumed. Here $p_{j}=n T_{j}, p$ is the total pressure. The $\Gamma_{j}$ 's are the ratios of the specific heats. The large $\kappa_{\| j}$ is accurately modeled using the artificial sound method.[7]

(a)

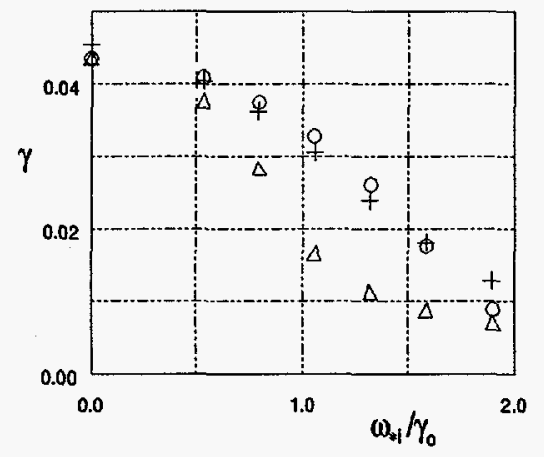

(b)

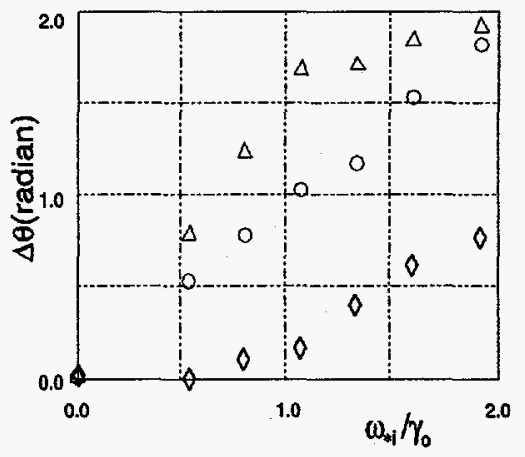

Figure 4: (a)Stabilization of the $m=1, n=1$ resistive mode compared to the analytic dispersion relation. Crosses represent the analytic growth rates, circles the MH3D-T results for equilibrium profiles $p_{e}=p_{i}$. Triangles show the MH3D$\mathrm{T}$ case with $p_{e}=0$, illustrating the destabilizing effect of $\omega_{* e}$. (b)Direction of the $m=1, n=1$ plasma kink flow relative to the reconnection X-point in a poloidal cross section. Circles represent the ion fluid flow $\mathbf{v}_{i}$ and diamonds the guiding center velocity $\mathbf{v}$ for equilibrium $p_{e}=p_{i}$. Triangles represent $\mathbf{v}_{i}$ for $p_{e}=\mathbf{0}$.

The code has been benchmarked against analytic theory for the diamagnetic stabilization of the $m=1, n=1$ mode in a cylinder. The analytic dispersion relation was solved numerically [8] for a narrow reconnection layer. A uniform resistivity $\eta=S^{-1}=3.24 \times 10^{-5}$ and toroidal plasma beta $\beta_{o}=0.067$ at the center of the plasma was used. Viscosity and thermal and particle diffusion were 
small compared to the resistivity. $q$ varied from $q_{o}=1.1$ to $q_{a}=5$, with $q=2$ at $r / a=0.63$. In the first case, it was assumed that $p_{e}=p_{i}$ in equilibrium. The results are shown in Fig. 4(a), where the growth rate $\gamma$ is plotted against the diamagnetic parameter $\omega_{* i} / \gamma_{o}$. Crosses represent the analytic results, and circles the numerical results. Good agreement is found over a wide range of growth rate. The destabilizing effect of the electron diamagnetic frequency $\omega_{* e}$ at fixed total $\beta$ is seen by comparing the case $p_{e} \equiv 0$ (triangles).

The ion diamagnetic stabilization has a simple physical interpretation.[4] The direction of the outward kink motion of the plasma inside the $q=1$ surface rotates poloidally away from the reconnection X-point as $\omega_{* i} / \gamma_{0}$ increases, up to approximately $\pi / 2$. This reduces the efficiency of the mode drive, and slows the rate of reconnection. The relative angle $\Delta \theta$ versus $\omega_{* i} / \gamma_{o}$ is plotted in Fig. 4 (b). For the case $p_{e}=p_{i}$ the angle of the radial ion fluid flow $v_{i r}$ is given by circles and the particle flow $v_{r}$ by diamonds. The electron diamagnetic drift $\omega_{* e}$ exerts its destabilizing effect by resisting the rotation of the kink. When $p_{e} \equiv 0$, flow rotation and mode stabilization develop much faster (triangles show $v_{i r}$ ).

The $M H 3 D-T$ code has been used to study the rotation of linear and nonlinear resistive modes in a torus. The linear mode rotates in the $\omega_{* e}$ direction if $p_{e}$ is comparable to $p_{i}$, and in the $\omega_{* i}$ direction if $p_{e}$ is small (in the electron guiding center frame). Nonlinearly however, the magnetic island rotation is quite different. From Eq. 9, one can see that finite size islands have to be stationary (except for a slow speed proportional to $\eta$ ) in the electron guiding center frame, if $\nabla_{\|} p_{e}$ is small. This is because the $\mathrm{X}$-point has to move with $\mathbf{v}_{\perp}$ from the frozen-in flux condition for $\eta=0$. Any rotation speed on the order of a fractional power of $\eta$ would require a fast reconnection process. This requires a large free energy which is absent near the saturation of the mode. Simulation results agree with this reasoning. For $\nabla_{\|} p_{e} \neq 0$, islands rotate with $\omega_{* e}$. However, $\nabla_{\|} p_{e} \propto n \nabla_{\|} T_{e}+T_{e} \nabla_{\|} n$ and $\nabla_{\|} T_{e}$ becomes negligibly small in the electron transit time scale, while $\nabla_{\|} n$ becomes small in the sound wave time scale(as also seen in Ref. 9 and 10 using reduced equations). If the plasma rotation is very fast, $\nabla_{\|} n$ can remain significant due to centrifugal force, but for a realistic rotation speed it can be neglected. In the simulation with TFTR parameters, it takes about 100 to 1000 sound wave transit times for $\nabla_{\|} n$ to become small for islands of a few $\mathrm{cm}$, because of the proximity of low mode number rational surfaces. This gives 0.1 to $1 \mathrm{msec}$ for $\nabla_{\|} n$ flattening. $\left(\nabla_{\|} T_{e}\right.$ flattening is much faster.) Since magnetic islands in tokamaks can form as fast as $\sim 0.1 \mathrm{msec}$ through a fast reconnection precess, an experimental measurement of the slowing down of the magnetic island rotation(in the electron guiding center frame) due to the flattening of $\nabla_{\|} n$ may be feasible.

\section{Particle/MHD hybrid simulation}

To model the nonlinear interaction of energetic particles with MHD waves, a hybrid particle/MHD model had been developed.[11] The plasma is divided into two parts: the bulk plasma, which contains the thermal electrons and ions, and the energetic hot ions. The bulk plasma is described by the ideal MHD equations, whereas the hot ions are described by the gyrokinetic equations[12]. The particle part can be coupled to the bulk plasma part through one of two almost equivalent, accurate coupling schemes, the pressure coupling and current 
coupling. In the pressure coupling scheme, the hot particle pressure tensor $\mathrm{P}_{h}$ is coupled to the bulk plasma momentum equation:

$$
\rho_{b} \frac{d \mathbf{v}_{b}}{d t}=-\nabla P_{b}-\left(\nabla \cdot P_{h}\right)_{\perp}+\mathbf{J} \times \mathbf{B}
$$

In the current coupling scheme, the hot particle current density $\mathbf{J}_{h}$ and charge density $q_{h}$ are coupled to the bulk plasma momentum equation:

$$
\rho_{b} \frac{d \mathbf{v}_{b}}{d t}=-\nabla p_{b}+\left(\nabla \times \mathbf{B}-\mathbf{J}_{h}\right) \times \mathbf{B}+q_{h} \mathbf{v}_{b} \times \mathbf{B}
$$

where the subscript $b$ denotes the bulk part and the subscript $h$ denotes the hot ion component. The last term of Eq. 16 can be thought of as the subtraction of the $\mathbf{J} \times \mathbf{B}$ force on the electrons whose density is the same as the hot particles. This term cannot be neglected because the $\mathbf{E} \times \mathbf{B}$ drift can be comparable to the perturbation of the magnetic drift of the hot particles.

Equation 15 or Eq. 16, together with the other MHD equations form the MHD part which is advanced in time using particle quantities given by the particle part. The new $\mathbf{E}$ and $\mathbf{B}$ are in turn used by the particle part to advance the particle quantities in time. The model is fully self-consistent, including nonlinear Landau damping and other hot particle interactions with MHD waves, and the nonlinear MHD mode coupling.

Using the particle/MHD hybrid $M H 3 D-K$ code we have found that wave particle trapping is the dominant mechanism for the nonlinear TAE saturation.[13] In this work, the pressure coupling scheme was employed. The "double trajectory method" was used to reduce the simulation noise in the linear regime. In this method, two sets of particles are used, one following the equilibrium field and the other the total electromagnetic field. The hot particle pressure tensor $P_{h}(t)$ is replaced by $\left(P_{h}(t)-P_{h, 0}(t)\right)+P_{h, 0}(0)$ where $P_{h, 0}$ is evaluated from the equilibrium orbits. The advantage of the double trajectory method is that it can be applied self-consistently to any $3 \mathrm{D}$ equilibrium with an arbitrary distribution of particles including a delta function in velocity space. The disadvantage is that it is only valid for the linear regime.

The $\delta f$ method of noise reduction[15], on the other hand, also applies in the nonlinear regime. However, because of difficulties, it has not been applied in a self-consistent manner to a $3 \mathrm{D}$ electromagnetic problem with a self-consistent equilibrium. We have devised a scheme for a self-consistent $\delta f$ method for such cases, and implemented it in the MH3D-K code. The new linear results agree closely with the double trajectory method results, while improved nonlinear saturation results have been obtained which still give the same conclusion as obtained before in Ref. 13. The self-consistent $\delta f$ method and MH3D-K simulation results of TAE saturation will be described at this conference by $\mathrm{Fu}$ et al. in a TAE mode paper.

\section{Unstructured mesh code}

As demonstrated in fluid dynamics research, the most efficient way to represent general geometric effects is to use an unstructured numerical mesh. Finite element, unstructured mesh methods are now just beginning to be used in MHD computations. Unstructured meshes offer two important advantages. They may 
be fitted to complicated geometries. This is necessary for simulations of divertor tokamaks. $[16,17]$ The second advantage is the ability to introduce localized mesh refinement. For example, extra vertices may be added at a magnetic X-point.

The meshpoints of the unstructured mesh are the vertices of triangles (see Fig. 5(a)), located at points $\vec{r}_{i}$. The most convenient basis functions are piecewise linear "tent" functions, $\lambda_{i}(\vec{r})$, which are nonzero at a vertex common to several triangles, and which vanish at all other vertices.

The variables in the MHD equations are represented as a sum over poloidal finite element basis functions and toroidal Fourier harmonics. We use a mixed method in which the variables to be expanded in basis functions include the electrostatic potential $\phi$, magnetic flux $\psi$, toroidal vorticity $W$ and toroidal current $C$. The MHD equations are discretized with a zero residual Galerkin approach, in which the equations are multiplied by a basis function $\lambda_{j}$ and integrated over the domain. This gives a set of sparse matrix equations, in which the differential operators become sparse matrices involving integrals of the basis functions and their derivatives. These can be done analytically. In the Laplacian, integration by parts is used to avoid having to take second derivatives. The primary matrices appearing in the discrete equations are the mass matrix $\mathbf{M}$, the stiffness matrix $\mathbf{S}$, and the Poisson bracket tensor $\mathbf{P}$, defined by

$$
\begin{aligned}
M_{i j} & =\int \lambda_{i} \lambda_{j} d^{2} x \\
S_{i j} & =-\int \nabla \lambda_{i} \cdot \nabla \lambda_{j} d^{2} x \\
P_{i j k} & =\int \lambda_{i} \nabla \lambda_{j} \times \nabla \lambda_{k} \cdot \hat{z} d^{2} x
\end{aligned}
$$

Both the stiffness and mass matrices are symmetric. The Poisson bracket is anti-symmetric under the exchange of any two indices. This assures that some of the most important integral relations satisfied by the differential equations are preserved by the finite element discretization. This includes conservation of energy and magnetic flux in the absence of dissipation. The matrices are very sparse, having nonzero elements only between those vertices connected by the side of a triangle.

Although the use of the stiffness matrix causes no problems when the electrostatic potential $\phi$ is calculated from the toroidal vorticity $W$, there is a loss of accuracy and even convergence when the toroidal current $C$ is calculated from the poloidal flux $\psi$ using the stiffness matrix. Convergence is restored by calculating the current in two steps: first calculating the poloidal magnetic field components from $\psi$, and expanding the result in basis functions; then taking the toroidal component of the curl and again expanding in basis functions. This is equivalent to using a larger stencil for the current calculation, which preserves the necessary symmetries for energy conservation [18].

The finite element unstructured mesh discretization has been incorporated into $M H 3 D^{++}$with an object oriented approach. A benefit of object oriented programming is that the objects are like black boxes whose inner workings are hidden from and protected from the user. They can be linked with other code in a simple way, without having to be concerned with details of their inner workings. The unstructured mesh objects, called MeshObject, generate an unstructured mesh, create all necessary auxiliary arrays, and produce the sparse matrices which implement differential operators including gradient, curl, and 

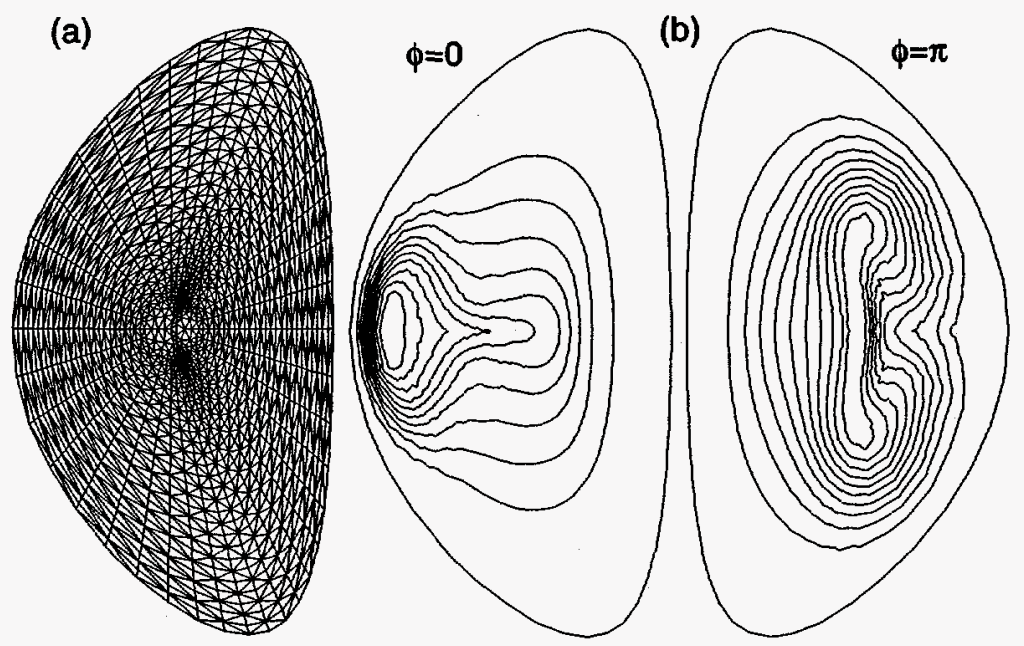

Figure 5: (a)An unstructured mesh for an ITER-like geometry. Note that the mesh has no origin. (b)Pressure contours resulting from nonlinear development of a pressure driven mode after a pellet injection.

divergence, as well as various Poisson solvers based on the Incomplete Cholesky Conjugate Gradient method.

An important feature of this approach is that most of the MH3D code is retained. The user has the option of linking the code in the standard way, to produce a code that runs as a finite difference/Fourier code; or linking with MeshObject, to give an unstructured mesh. This allows direct benchmarking of the two versions against each other. Equilibrium and stability calculations using the two versions have been compared, and there is good agreement.

As a nontrivial example of the use of the $M H 3 D^{++}$code, we consider pellet injection into an ITER like tokamak. The pellet is assumed to rapidly heat and form a large pressure perturbation, which is poloidally and toroidally localized. The peak pressure of the perturbation is 0.25 of the peak pressure in the tokamak, which has $4 \%$ peak $\beta$. The initial state consists of an equilibrium, on which the pellet perturbation is superposed. A pressure driven instability develops and undergoes a large distortion as shown in Fig. 5(b). The implication of this result is currently under study.

\section{Conclusion}

The M3D(Multi-level 3D) tokamak simulation project aims at the simulation of tokamak plasmas using a multi-level tokamak code package. Several current applications are presented; high- $\beta$ disruption studies in reversed shear plasmas using the MHD level $M H 3 D$ code, $\omega_{* i}$ stabilization and nonlinear island rotation studies using the two-fluid level $M H 3 D-T$ code, studies of nonlinear saturation of TAE modes using the hybrid particle/MHD level $M H 3 D-K$ code, and unstructured mesh $M H 3 D^{++}$code studies. In particular, three internal mode disruption mechanisms are identified from simulation results which agree well with experimental data.

The successful applications of these MHD and Extended-MHD codes support the premise of the M3D project that a multi-level simulation code is necessary for the study of tokamaks, where the complex phenomena can be modeled with 
various levels of realism. It is also a hopeful sign that this step by step path could eventually lead to a comprehensive tokamak simulation code which would include most of the relevant physics and also allow various option levels in complexity of physics and geometry.

\section{Acknowledgements}

This work was supported by the United States Department of Energy under Contract DE-AC02-76-CHO-3073, Contract DE-FG02-91ER54109, and Grant DE-FG02-86ER53223.

\section{References}

[1] W. Park et al., Phys. Rev. Lett. 751763 (1995).

[2] Z. Chang et al., PPPL Report-3191 (1996), to appear in Phys. Rev. Lett.

[3] W. Park D. A. Monticello, E. Fredrickson, K. McGuire, Phys. Fluids B 3, 507 (1991).

[4] L.E. Sugiyama and W. Park, M.I.T. Research Laboratory of Electronics Report PTP-96/1 (1996), submitted to Phys. Plasmas.

[5] R.D. Hazeltine and J.D. Meiss, Phys. Rep. 121 (1985) 1; Plasma Confinement, (publ. Addison-Wesley, Redwood City CA, 1992).

[6] S.I. Braginskii, in Reviews of Plasma Physics, ed., Leontovich, M.A., Vol. 1, p. 205.

[7] W. Park, D.A. Monticello, H. Strauss, and J Manickam, Phys. Fluids 29, 1171 (1986).

[8] S.M. Migliuolo, private communication.

[9] D. Biskamp, Nucl. Fusion 19 (1979) 777.

[10] B.D. Scott, A.B. Hassam, and J.F. Drake, Phys. Fluids 28 (1985) 275.

[11] W. Park et al., Phys. Fluids B 4, 2033 (1992).

[12] W. W. Lee, J. Comput. Phys. 72, 243 (1987)

[13] G. Y. Fu and W. Park, Phys. Rev. Lett. 741594 (1995).

[14] H. L. Berk, B. N. Breizman, and H. Ye, Phys. Rev. Lett. 68,3563 (1992).

[15] S.E. Parker and W.W. Lee, Phys. Fluids B 5, 77 (1993).

[16] H. Strauss and D. W. Longcope, submitted to J. Comp. Phys. (1996).

[17] H.R. Strauss, Phys. Plasmas 2, 1229 - 1235, (1995).

[18] H. Strauss, Phys. Plasmas, to appear (1996). 